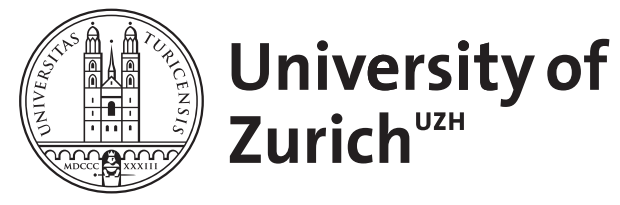

Zurich Open Repository and Archive

University of Zurich

Main Library

Strickhofstrasse 39

CH-8057 Zurich

www.zora.uzh.ch

Year: 2014

Recurrent spontaneous coronary artery dissection in a 46-year-old woman

Amoruso, Marco ; Moccetti, Tiziano ; Pedrazzini, Giovanni B

Posted at the Zurich Open Repository and Archive, University of Zurich

ZORA URL: https://doi.org/10.5167/uzh-106254

Journal Article

Originally published at:

Amoruso, Marco; Moccetti, Tiziano; Pedrazzini, Giovanni B (2014). Recurrent spontaneous coronary artery dissection in a 46-year-old woman. Cardiovascular Medicine:269-272. 


\section{Recurrent spontaneous coronary artery dissection in a 46-year-old woman}

\section{A case report and literature review}

Marco Amoruso, Tiziano Moccetti, Giovanni Pedrazzini

Department of Cardiology, CardioCentroTicino, Ticino, Switzerland

\section{Summary}

Spontaneous coronary artery dissection is a rather rare cause of chest pain. Recurrent spontaneous dissection is even more uncommon and the extant literature reports very few cases. We describe the case of a 46-yearold woman, who presented two spontaneous coronary dissections in different coronary territories with a three-year lapse. An invasive treatment at first and a conservative one the second time were performed successfully. Epidemiology, pathogenesis, clinical features and diagnosis are also discussed.

Key words: recurrent spontaneous coronary artery dissection; acute coronary syndrome

\section{Introduction}

About 500 cases of spontaneous coronary artery dissection (SCAD) have been reported in the medical literature. Recurrent spontaneous dissection is even more uncommon: we describe the unusual case of a 46-yearold woman, presenting a two-step spontaneous coronary dissection. The first episode occurred in the middle part of the left circumflex artery (LCx), and a multiple stent implantation was performed. After thirty-four months, the second event involved the distal segment of the right coronary artery (RCA) and the posterior lateral branch: a conservative treatment decision was made and a spontaneous recanalisation was observed at follow-up.

\section{Figure 1}

November 2003 coronary angiography.

A Fresh, long dissection on middle LCX artery (arrows). Normal LAD artery.

B Middle $\mathrm{LCX}$ artery $\mathrm{PCl}$ and stent implantation (arrows).

C Normal RCA.

$\mathrm{LAD}=$ left anterior descending artery; $\mathrm{LCX}=$ left circumflex artery; $\mathrm{RCA}=$ right coronary artery; $\mathrm{PCI}=$ percutaneous coronary intervention.

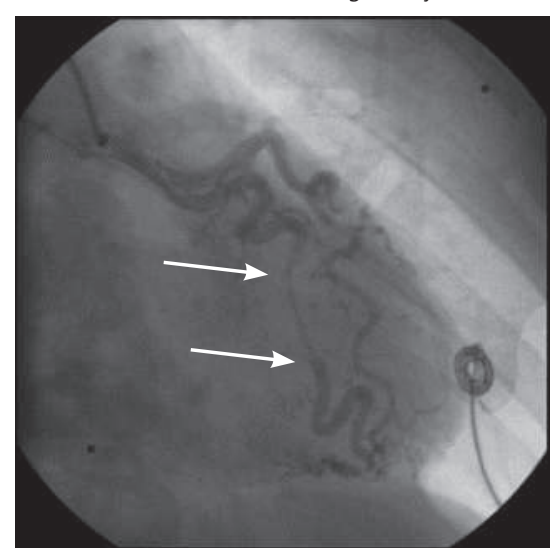

A

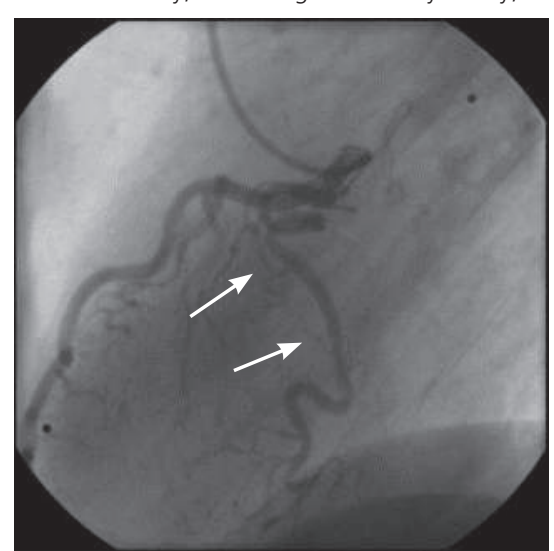

B



C
Funding / potential competing interests:

No financial support and no other potential conflict of interest relevant to this article were reported.
Correspondence:

Marco Amoruso, MD

Department of Cardiology, CardioCentroTicino

Via Tesserete 48

$\mathrm{CH}-6900$ Lugano

Switzerland

marco.amoruso[at]cardiocentro.org 


\section{Case presentation}

In November 2003, a 46-year-old woman, active smoker, with mild hypertension and a family history of cardiovascular disease, reported a sudden onset of pressurelike substernal chest pain, radiating to both arms: while being transported to the emergency department by ambulance, a cardiac arrest occurred with ventricular fibrillation as the first rhythm established. Cardiopulmonary resuscitation and direct current shock were performed and the return of spontaneous circulation was achieved. In the emergency department ECG showed regular sinus rhythm, $65 \mathrm{bpm}$, and ST depression in $\mathrm{V}_{2}-\mathrm{V}_{6}$ leads. The coronary angiography showed a long (around $25 \mathrm{~mm}$ ) subtotal stenosis and narrowed vessel aspect (TIMI I) in the middle part of the tortuous LCx, with a small radiolucent defect in the proximal segment of the lesion, suggestive features of spontaneous dissection, without any other detectable coronary lesion. The patient underwent percutaneous coronary intervention (PCI) and three drug-eluting stents (Taxus-paclitaxel) were successfully implanted (fig. 1), resulting in restoration of TIMI III flow and resolution of symptoms. The patient improved and was discharged on double antiplatelet therapy for twelve months (Aspirin ${ }^{\circledR}$ and clopidogrel), beta-blocker and statin. Follow-up coronary angiography at three months demonstrated patency of the stents, without evidence of residual disease, and her medical treatment was confirmed. Thirty-four months later, in August 2006, the patient was admitted with unstable angina, positive troponin and T-wave inversion in precordial leads $\left(\mathrm{V}_{2}-\mathrm{V}_{5}\right)$. The coronary angiography showed a fresh and long $(>20 \mathrm{~mm})$ dissection of the distal RCA propagating to the posterior lateral branch without any sign of disease in the other vessels (fig. 2). Once again the proximal segment of the lesion presented a tiny radiolucent line. In light of the unfavourable location of the lesion, its extension, the small size of the target vessel, and the symptoms easily managed with nitrates, a conservative treatment was deemed necessary. The patient had a favourable clinical course and discharge occurred three days later, on aspirin and clopidogrel for 3 months, beta-blocker and statin. In the follow-up the patient remained almost asymptomatic. Forty-eight months later, in August 2010, because of recurrence of chest pain and inconclusive Echo-stress test, a new coronary angiography was performed. It showed a spontaneous recanalisation of the previously dissected RCA and posterior lateral branch, with a small persisting lesion (fig. 3); no sign of injury was found in the other vessels. Aspirin was confirmed at discharge. Since then, the patient is still on aspirin and beta-blocker, and completely asymptomatic at the follow-ups, last one performed in February 2014.

\section{Discussion}

SCAD usually occurs in the female population 8-9 times more often than in the male population. The mean age referred is between 35 and 46 years [1]. Within the angiographies performed for acute coronary syndromes or stable angina, prevalence of SCAD varies from 0.2 to $1.1 \%$ [1]. Nevertheless, women younger than 50 years old showing ST elevation acute coronary syndrome are believed to have an incidence of around $10 \%$ [1]. Recurrent SCAD is even more rare. Because of limited clinical studies, the incidence of recurrent SCAD is difficult to estimate: in a retrospective cohort of 87 patients with SCAD, recurrence at forty-seven months occurred in $17 \%$, mainly in previously unaffected coronary vessels despite risk factor management [2]. Moreover, all the patients with recurrence were female and a connection between recurrence and noncoronary fibromuscular dysplasia (FMD) was described [2]. SCAD is generally related to atherosclerosis or the peri-partum period. The pathogenesis is not fully understood. The rupture of atherosclerotic plaque or haemorrhage from weakened vasa vasorum may induce a dissection plane in the layers of the arterial
Figure 2

August 2006 coronary angiography.

A Normal LAD and normal LCX artery, with patency of the previous stents on middle $L C X$.

B Fresh, long dissection of distal RCA and posterior lateral branch (arrows).

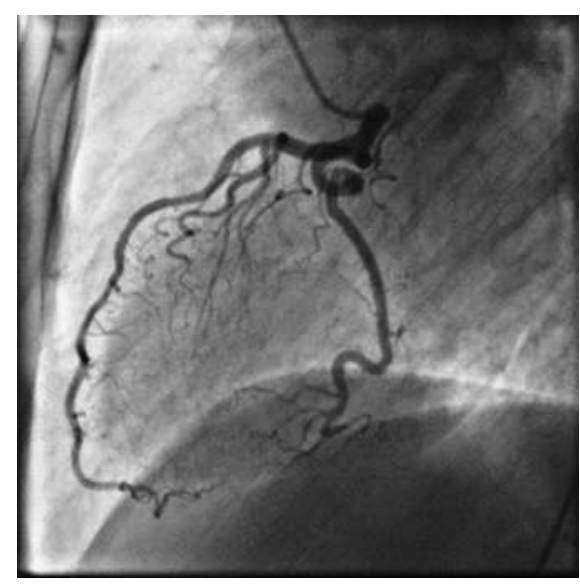

A

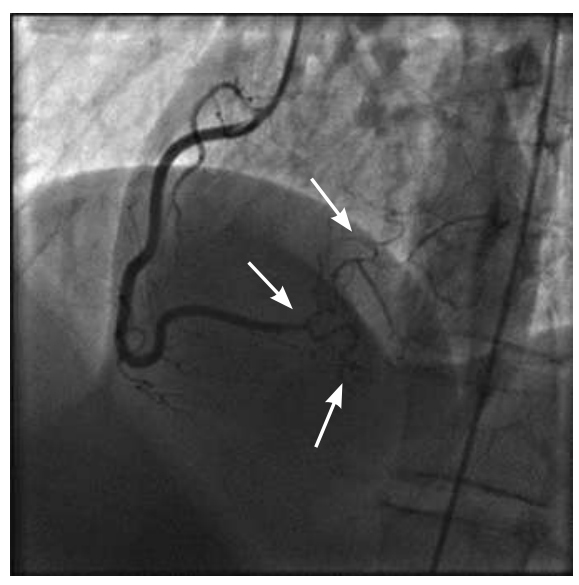

B 
Figure 3

August 2010 coronary angiography.

A RCA spontaneous recanalisation with a small persisting lesion on the posterior lateral branch (arrows)

B Normal LAD and LCx artery with patency of the previous stent on $\mathrm{LCX}$

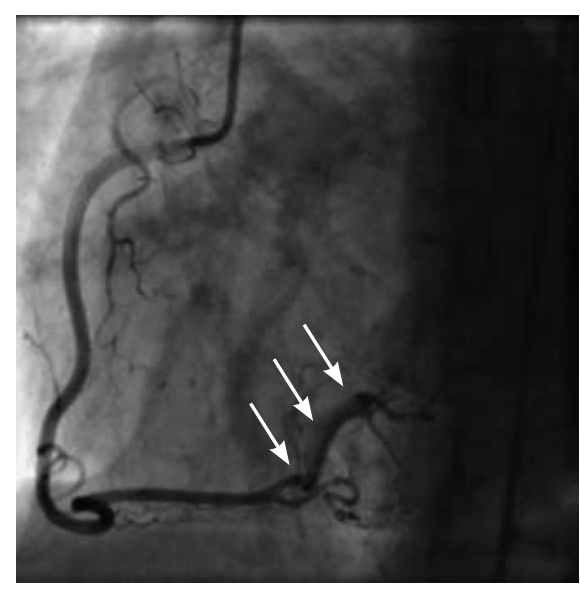

A

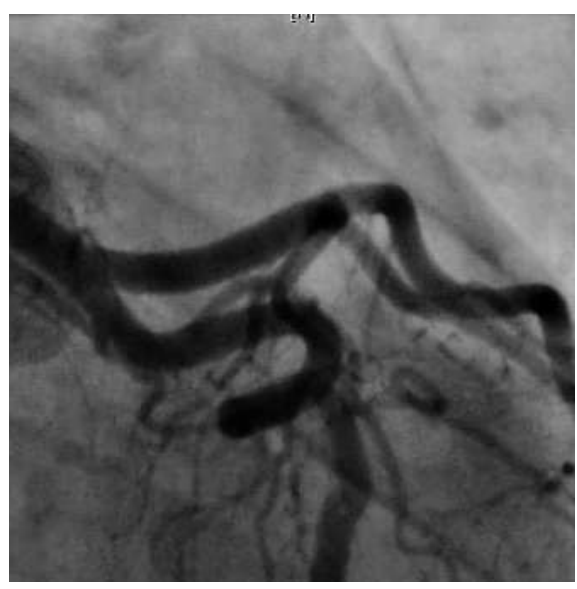

B wall. The blood collecting in the false lumen may lead to dilating haematoma and compression of the true lumen, resulting in restricted coronary blood flow, acute coronary syndrome or even sudden death [2]. On the other hand, during pregnancy and early post-partum period, progesterone-induced weakening of vessel walls, hypercoagulability and elevated blood pressure with increased shear stress might lead to dissection [3]. Moreover, intense emotional situations may generate coronary dissection [1]. Many others entities have been linked to SCAD: excessive exercise, spasm and spastic agent (e.g., cocaine), connective tissue disorders (Marfan's syndrome, type IV Ehlers-Danlos syndrome, systemic erythematous lupus) and vasculitis (polyarteritis nodosa, Kawasaki disease and eosinophilic arteritis) [2]. The fibromuscular dysplasia, often in the setting of medial fibroplasia (80-90\%), seems to play an important role in patients with non-atherosclerotic SCAD. Saw et al. found that most of these patients (86\%) have FMD in at least one non-coronary territory (renal, iliac, cerebral) suggesting a link between an underlying coronary FMD and SCAD [4]. Regarding the overall angiographic distribution of $\mathrm{SCAD}$, a review of the literature shows LAD or multi-vessel involvement in the vast majority of the cases, RCA less frequently involved (predominantly in men) and LCx artery, left main stem and obtuse marginal seldom mentioned [5]. SCAD presentation ranges from stable angina to acute myocardial infarction (STEMI/NSTEMI) with cardiogenic shock or sudden death [5]. Tweet et al. found that $14 \%$ of their cohort experienced ventricular fibrillation or tachycardia requiring defibrillation [2]. The performed angiograms showed a subtotal stenosis of the vessel with narrowed aspect and a radiolucent defect suggestive of endothelial injury in the proximal segment of the lesion. In the literature, a thin radiolucent line representing the intimal flap as starting lesion is often described. Less frequently, coronary vessels appear narrowed or occluded because of a medial haematoma leading to compression of the true lumen [6]. Recently, intravascular diagnostic techniques have allowed a better resolution of the vessel wall injuries, like the intimal flap, and the true and false lumen: the intravascular ultrasound can represent the extent of the disease even in large vessels, determining the aspect and size of the haematoma, and improving the precision of PCI. As a result of its high resolution, the optical computed tomography may describe the intimal tear and the inner layers of the media, providing information about the healing process of the coronary wall [7]. The critical clinical presentation of the first episode, together with angiographic finding of dissection in a major vessel with reduced flow (TIMI I), led us to a percutaneous coronary intervention and stent implantation. By contrast, the haemodynamic stability, regression of angina with nitrates and small size of the dissected vessel let us conservatively treat the patient three years later. Currently, no specific guideline exists about treatment in SCAD. Factors that may condition the treatment strategy include site of dissection, vessels involved and haemodynamic parameters [5]. Conservative therapy seems appropriate in stable patients with mid- or distal SCAD, limited dissection at angiogram and normal flow in the affected artery $[1,2]$. It consists principally of beta-blocker and nitrates; antiplatelet (aspirin, clopidogrel, glycoprotein IIb/IIIa inhibitors) and anticoagulant (heparin) therapy are accepted to decrease thrombus formation in the false lumen even if the possibility of bleeding increase and propagation of dissection is reported [2]. On the other hand, PCI is considered in a major vessel disease or ongoing ischaemia pattern but it may propagate the dissection or cause stent deployment into the false lumen with abolition of coronary flow $[2,5]$. Coronary artery bypass graft is generally reserved to left main stem or multivessel involvement [5]. Despite the different presentations, the patient made a good recovery each time. Unexpectedly, the coronary angiography performed in 
2010 showed almost complete healing of the previously dissected distal RCA and confirmed LCx patency. In our case, both invasive and conservative treatments, closely related to clinical presentation and angiographic findings, have shown to be effective options. The presentation of SCAD as acute coronary syndrome is related to an initial morbidity with a mean value of in-hospital mortality of around 3\% [1]; as a long-term outcome, Tweet et al. found in their cohort of patients a 1- and 10-year mortality rate of 1.1 and $7.7 \%$, respectively, and a 10-year rate of major adverse cardiac events (MACE) of 47.4\% [2]. Alfonso et al. reported 45 consecutive patients with SCAD initially conservatively managed and with revascularisation restricted to those with ongoing ischaemia: at follow-up, the 3-year event-free survival was $92 \%$ [7].

\section{Conclusions}

Our case report of recurrent SCAD after 3 years in a 46-year-old woman warns us that dissection is a differential diagnosis in the assessment of chest pain, especially in female patients. Presentation may span the clinical spectrum from stable angina to acute myocardial infarction and all the coronaries may be involved: a careful, individual assessment is critical. Restoration of normal coronary blood flow can be achieved with medical treatment only or PCI depending on the clinical presentation and the angiographic evaluation. Because of the long-term prevalence of MACE and the unpredictability of recurrence of the disease, a longterm follow-up in SCAD is recommended in our opinion.

\section{References}

1 Vanzetto G, Berger-Coz E, Barone-Rochette G, et al. Prevalence, therapeutic management and medium-term prognosis of spontaneous coronary artery dissection: results from a database of 11,605 patients. Eur J Cardiothorac Surg. 2009;35:250-4.

2 Tweet MS, Hayes SN, Pitta SR, et al. Clinical features, management, and prognosis of spontaneous coronary artery dissection. Circulation. 2012;126:579-88.

3 Appleby CE, Barolet A, Ing D, et al. Contemporary management of pregnancy-related coronary artery dissection: a single - center experience and literature review. Exp Clin Cardiol. 2009;14:e8-e16.

4 Saw J, Ricci D, Starovoytov A, et al. Spontaneous coronary artery dissection: prevalence of predisposing conditions including fibromuscular dysplasia in a tertiary center cohort. JACC Cardiovasc Interv. 2013;6(1): 44-52.

5 Sheikh AS, O'Sullivan M. Pregnancy-related Spontaneous coronary artery dissection: two case reports and a comprehensive review of literature. Heart Views. 2012;13(2):53-65.

6 Choi SW, Nam CW, Bae HJ, et al. Spontaneous coronary artery dissection diagnosed by intravascular ultrasound and followed up by cardiac computed tomography Korean J Intern Med. 2013;28(3):370-3.

7 Alfonso F. Spontaneous coronary artery dissection. New insights from the Tip of the Iceberg? Circulation. 2012;126(6):667-70. 